\title{
DESIGN OF A DYNAMIC MOCK-UP BENCH FOR TESTING ROBOTIC INTERVENTIONS
}

\author{
Luana Barbosa Pina Pereira \\ luana.barbosa.pina.pereira@cern.ch
}

Carlos Veiga Almagro, Luca Rosario Buonocore, Raul Marin Prades, Mario Di Castro and Alessandro Masi carlos.veiga.almagro, luca.rosario.buonocore, raul.marin.prades, mario.di.castro, alessandro.masi @cern.ch

\begin{abstract}
When a robotic intervention is required in hazardous facilities (e.g. particle accelerators or nuclear plants), it is commonly not possible to test the operation on-site in advance - a considerable challenge since robotic interventions usually require specific tasks for each location -, precluding the team from demonstrating the feasibility of the operation. It becomes mandatory to develop a particular mock-up for each operation, unsuitable for reusing it in future missions. To solve this problem, a general dynamic mock-up bench was designed, allowing to centre the testing of all remote handled tasks and to choose the best set of robots to perform them.
\end{abstract}

Keywords: Mock-ups, Robotic interventions, Telerobotics, Hazardous environments.

\section{INTRODUCTION}

In hostile environments, to decrease human exposure to hazards, Remote Handling (RH) tasks - the action of using a mechatronic device to execute remote-controlled operations - are often needed. At the European Organisation for Nuclear Research (CERN) [1], the Mechatronics, Robotics \& Operation (MRO) section is responsible for planning and safely performing $\mathrm{RH}$ operations.

MRO section maintains a pool of robots able to perform various tasks, e.g. disposing of dangerous items, doing reconnaissance and monitoring, transporting/positioning components and using sensors for several purposes (radiation and temperature mapping, etc). When a CERN's section requires a robotic intervention, $\mathrm{MRO}$ and the requesting section work together to design $\mathrm{RH}-$ friendly systems to be handled by MRO robots.

After the mechanical design process, it is essential to test the operation before performing on-site interventions. These tests are performed first virtually (CAD and CAE software and Virtual Reality simulation) and afterwards physically (mockup tests).
Physical tests are usually performed in MRO laboratory, allowing the team to demonstrate the feasibility of the operation, to perceive and solve design-related problems, to figure out the best position for environmental cameras, to test different hypotheses (e.g. the defect of a component), among other possibilities.

To assist this process, a general mock-up bench was conceived. The bench contains several modules that allow MRO robots to test all its features (e.g. cutting materials, plugging/unplugging connectors, fasten bolts, sense external impulses), allowing tests for several operations in the same intervention, reducing time for preparing a specific mock-up area for each test.

This work presents the process of general conception, consideration of restrictions, conceptual and detailed design of all subcomponents of the mockup bench.

\section{POOL OF ROBOTIC SYSTEMS}

CERN has a considerable and expanding pool of robots that can be divided in two groups according to their specifications:

- CERN-designed robots: CERN counts with several self-designed robots, mainly focused on modularity and adaptability: using a lifting stage to comply with a range of work heights, integrating several kind of tools, etc. Some of the most important CERN-designed robots are CERNBot 1.0 [2] and 2.0 (wheeled modular robots), CRANEBot (non-wheeled robot that performs upside-down tasks carried by an overhead crane - see Figure 1) and TIM [3](Train Inspection Monorail - see Figure 2 -, a robot designed for providing remotecontrolled actions in the Large Hadron Collider tunnel). The communication operator/robots is established via $\mathrm{WiFi}$ and $4 \mathrm{G}$ adapters, allowing, from the surface, to teleoperate them at the required scientific facilities/tunnels at CERN.

- Commercial robots: Telemax and Teodor are tele-operated robots designed by Telerob 


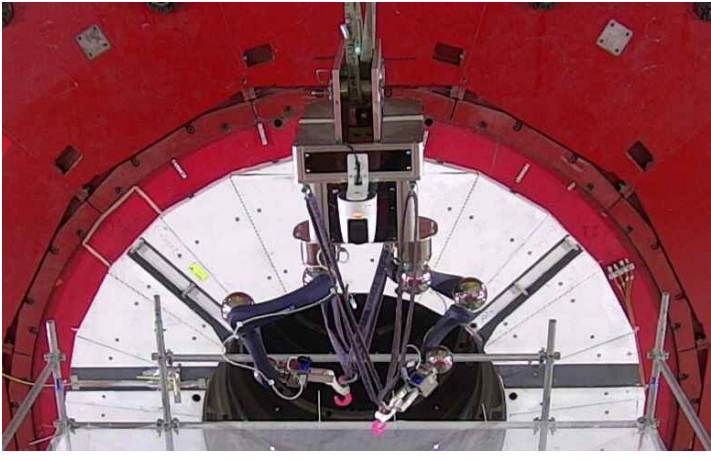

Figure 1: CRANEBot with dual arm configuration during an intervention at CMS experiment

GmbH [4]. Since Telemax is a highly accurate but light-weighted robot, and Teodor is a heavy robot with a high payload and low level of accuracy, they compensate each other disadvantages. Besides, for having tracked wheels, both robots are capable of climbing stairs and pass through obstacles of up to 50 $\mathrm{cm}$ of height. At last, the two robots require the presence of the operator close to the intervention area.

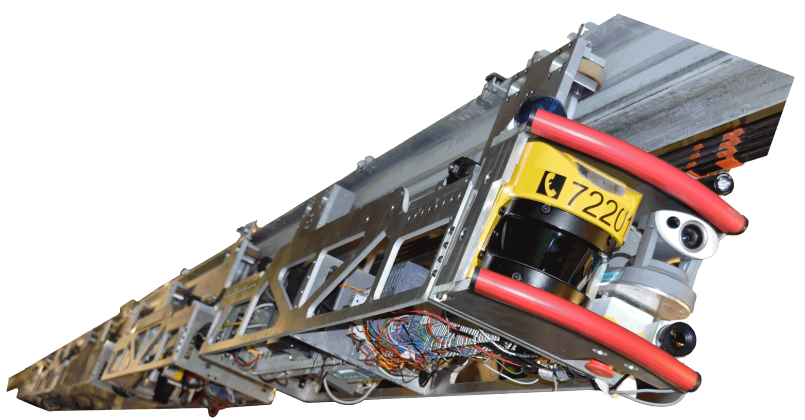

Figure 2: The Train Inspection Monorail hanging from the LHC's roof rail

\section{MECHANICAL DESIGN}

With the presented information on MRO robots, the design process of the bench was started. Its stages are detailed in the next subsections.

\section{$3.1 \quad$ REQUIREMENTS}

Based on the technical specifications of the robots and on MRO RH operations expertise, the scope of the design was set as following:

- The bench must bear several modules that allow MRO robots to test all its features, e.g. cutting materials, plugging/unplugging con- nectors, fasten bolts, sense external impulses (temperature, radiation, etc).

- These modules must be able to be operated from all work height levels of MRO robots, namely from 600 to $2000 \mathrm{~mm}$ from ground level.

- The bench must support a mass of approximately $100 \mathrm{~kg}$ (estimated overall mass of the modules attached to it), and its structure must withstand the maximum force exerted by Teodor, MRO's strongest robot (approximately $1000 \mathrm{~N}$ ), and its correspondent torque (dependent on the dimensions of the bench).

- The bench must be attached to the existing infrastructure on MRO laboratory (portrayed in Figure 3 alongside with the position chosen for the bench). To allow the operation of robots from the upper side (e.g. CRANEBot) and from the down side (e.g. CERNBot), no component can be placed above or under it.

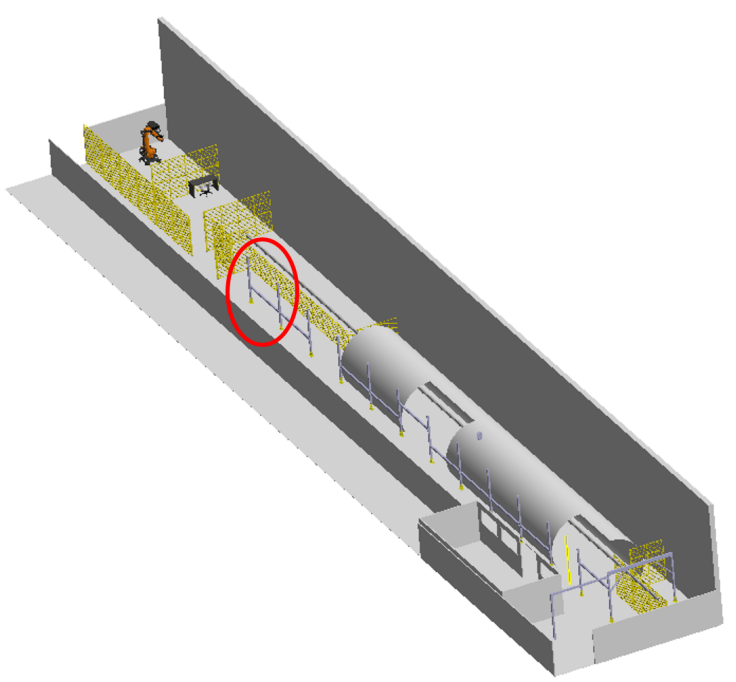

Figure 3: LHC mock-up at MRO laboratory

- At last, all modules must be accessed by both sides of the bench, allowing to test robots with a fix position, e.g. TIM.

\subsection{CONCEPTUAL DESIGN}

To fit the requirements set, possible concepts for the bench were conceived and analysed.

\subsubsection{Independent linear actuators}

Firstly, to comply with the requirement of having a work height range from $600 \mathrm{~mm}$ to $2000 \mathrm{~mm}$, a lifting mechanism is necessary. 
The first concept considered was linear actuators, lifting systems with integrated motor and linear driving mechanism. Commercial actuators as described can be seen in Figure 4.

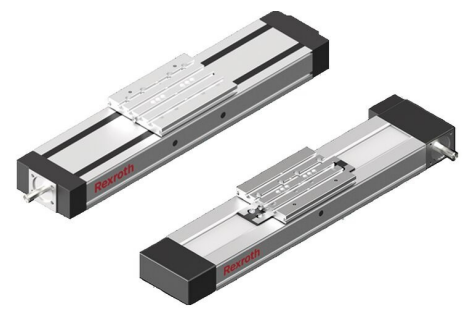

Figure 4: Commercial linear actuators [5]

One actuator attached to each side column would be necessary. Since they act independently, there would be no need for a mid-placed motor, abiding by the requirement of not placing any components below or above the bench. However, a control unit to coordinate the movement of the actuators would be necessary, increasing the complexity of the system.

\subsubsection{Screw jacks}

The second lifting concept analysed was screw jacks - rotating screws and linear guides positioned on each side of the bench. The system can be seen in Figure 5.

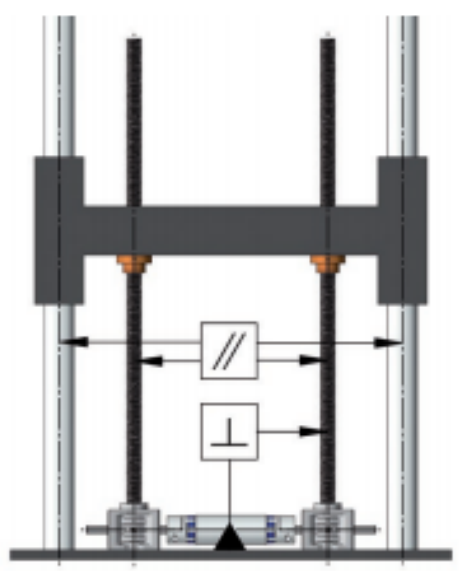

Figure 5: Screw jacks and guides layout $[6]$

This system supports the load and height range required. However, it depends on a single motor to actuate both screws - and the motor must be placed either under the bench, above it or on it. The first and second options do not comply with the requirement of not using the space directly under or above the bench.

The third option, placing the lifting motor on the bench, is at first possible: placed on the center of the bench, a motor is attached to two shafts that connect it to worm wheel gearboxes. However, by requirement, the table must also rotate to allow access to all modules from both sides. The tilt of the bench would make it impractical to connect the motor to the gearboxes by shafts - it would require an extra mechanism to prevent the motor from turning, once again increasing the complexity of the system.

\subsubsection{Steel cable}

To solve the problem of needing only one lifting motor but not placed either above, under or on the bench, a third concept was conceived.

It counts with a steel cable attached to one column, passing through the bench and ending on a hoist reel on the second column. In this way, only one lifting motor is necessary and it is placed outside of the upper or down operation space.

Pulleys are used to redirect the cable. Also, to keep the orientation of the bench, the laboratory columns were chosen as guides.

Since this solution solves the main requirements for the design and is less expensive than the previous analysed systems, it was selected as the lifting system concept.

\subsubsection{Harmonic drive}

Having decided on the steel cable concept for the lifting system, it is necessary to choose a rotary mechanism that does not interfere with it - a system with a hollow shaft, so the cable passes through the rotation axis of the bench.

The first concept considered was a harmonic drive, consisting of a synchronous servomotor and a gear unit. A commercial system as described can be seen in Figure 6.

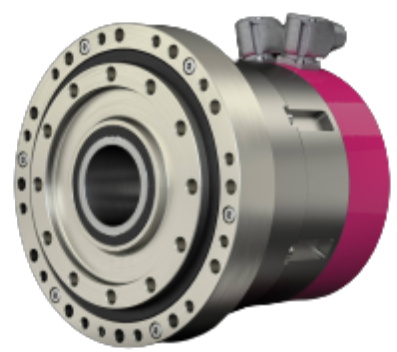

Figure 6: Commercial harmonic drive [7]

This concept delivers suitable power, although its price is elevated compared to more simple assemblies. More crucial to the project, however, is its capability of handling the torque generated by Teodor. The critical case would be Teodor ap- 
plying $1000 \mathrm{~N}$ on the edge of the bench. Based on the size of the modules to be attached to the bench, an estimation of its dimensions would be $600 \mathrm{~mm} \times 1800 \mathrm{~mm}$, resulting in a torque of 300 $\mathrm{N} / \mathrm{m}$. Therefore, the harmonic drive brake must be able to handle this load.

The torque to be handled by the brake motor is considerably large compared to the torque actually required to turn the bench, meaning the harmonic drive would be overdimensioned.

\subsubsection{Manual rotary system}

To be able to handle the critical case analysed previously without overdimensioning the design, a manual rotating system was conceived.

It is composed by fixed parts (a frame with holes spaced circularly), movable parts (perforated supports attached to the bench) and pins to connect both, allowing the bench to be tilted and locked.

Despite not being motorised and having only a finite number of angular positions, this solution does not rely on a motor brake to support external efforts, is more cost-effective and does not depend on a control unit. For these reasons, this concept was chosen as the rotary system of the bench.

\subsection{DETAILED DESIGN}

Having chosen the general concept of the bench, the detailed design of each subsystem was developed as following:

\subsubsection{Bench frame}

To allow attaching and repositioning the test modules, the bench is composed of standard aluminium profiles. As previously discussed, its general dimensions were set as $600 \mathrm{~mm}$ x $1800 \mathrm{~mm}$. In addition, the bench features horizontal and vertical-oriented profiles to facilitate the positioning of the modules, as shown in Figure 7.

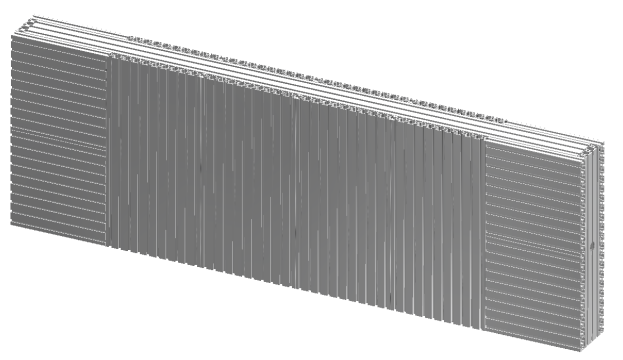

Figure 7: Bench frame

\subsubsection{Lifting mechanism}

Following the choice of a steel cable concept, instead of separately choosing a cable, a motor and a gearbox, it was opted for a commercial electric hoist commonly used for lifting goods. The system is composed of a reel for the cable, an electric motor and a control panel for operating the system. A commercial hoist as described can be seen in Figure 8.

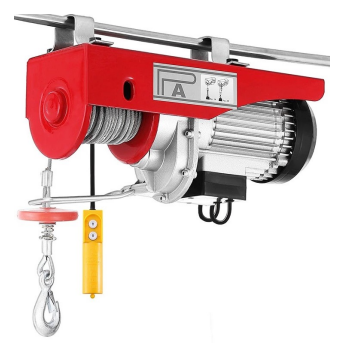

Figure 8: Commercial electric hoist [8]

The electric hoist will be rigidly fixed on the top part of the right column. The cable will be passed through the bench and fixed on the top of the left column. To change the orientation of the cable, two commercial pulleys were chosen. In addition, side supports were designed to hold the pulleys, with linear guides connecting them to the columns. Both the hoist and the linear guides were dimensioned to fit the design height and load range. The lifting system can be seen in Figure 9 .

It is noted that with heavy objects attached to the bench, the cable will expand elastically due to the load - therefore, the bench will have its position lowered. Nevertheless, since the bench will be operated statically, its height can be corrected using its remote control on the right side.

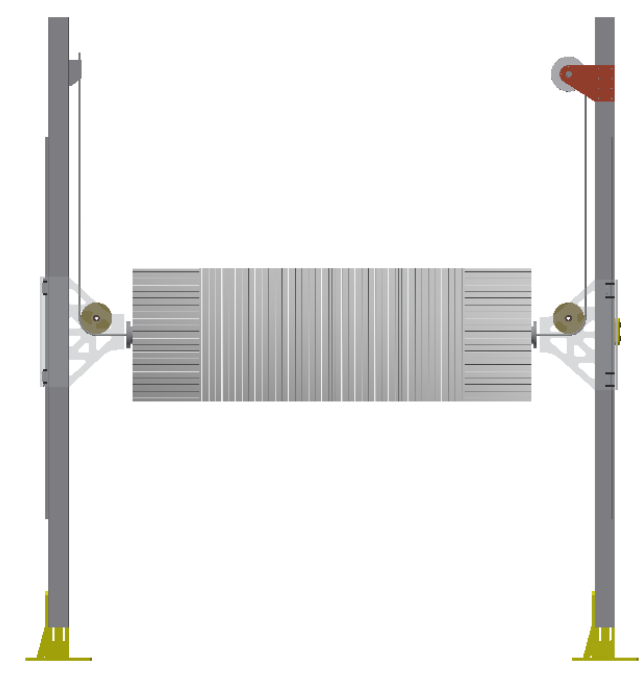

Figure 9: Lifting mechanism 


\subsubsection{Rotating mechanism}

To make sure the test modules can be accessed by all positions needed, the circular frame was set to have twenty equally spaced holes, as seen in Figure 10. It will be attached to the side supports of the lifting system.

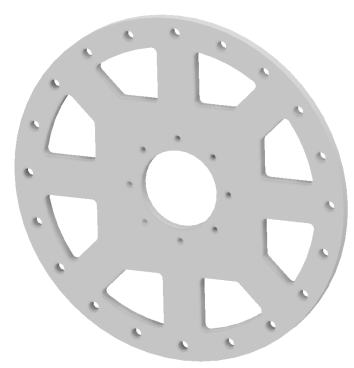

Figure 10: Circular frame

Linked to this frame there is a crossed roller bearing. This type of bearing is designed to support high loads, being suitable to handle Teodor's strength. The inner part of the bearing, on its turn, is connected to the aluminium profile of the bench frame.

At last, two perforated supports are attached to each side of the bench, allowing four fixation points. The supports, as well as the positioning of the bench, can be seen in Figure 11.
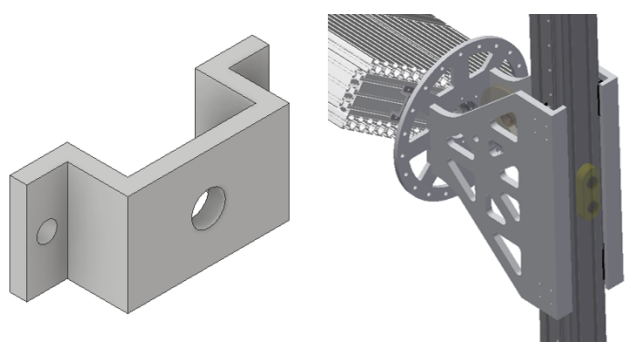

Figure 11: Rotating mechanism

Pins must be used to position and lock the bench. These pins and the side supports will handle the efforts the bench is submitted to, avoiding the need for a motor brake.

The final layout of the test bench can be seen in Figure 12.

\section{SIMULATION EXPERIMENTS}

The bench has been validated conceptually by integrating it into a realistic simulation server to test the way the robots can interact with it. In Figure 13, TIM robot can be seen facing the bench in order to perform a test with a robotic arm. Also, CERNBot mobile robotic platform is facing the

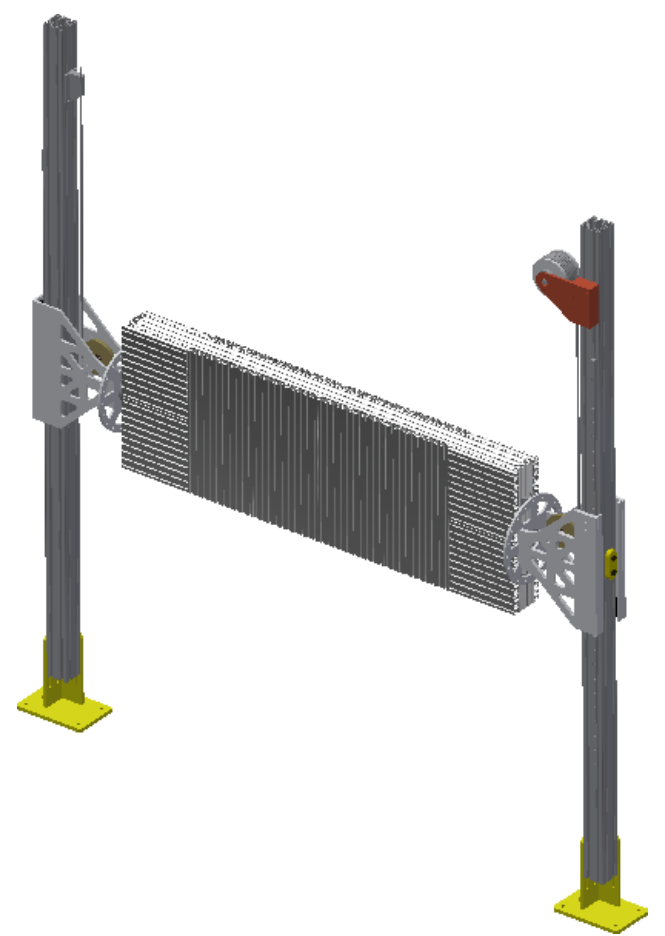

Figure 12: Final layout of the test bench

other side of the bench. By simulating interventions, it was proved that all robots can properly operate the bench within the height and rotational range set. The simulation experiments have been performed by using a simulation server which enables operators to get trained before facing a real intervention, as explained in [9].

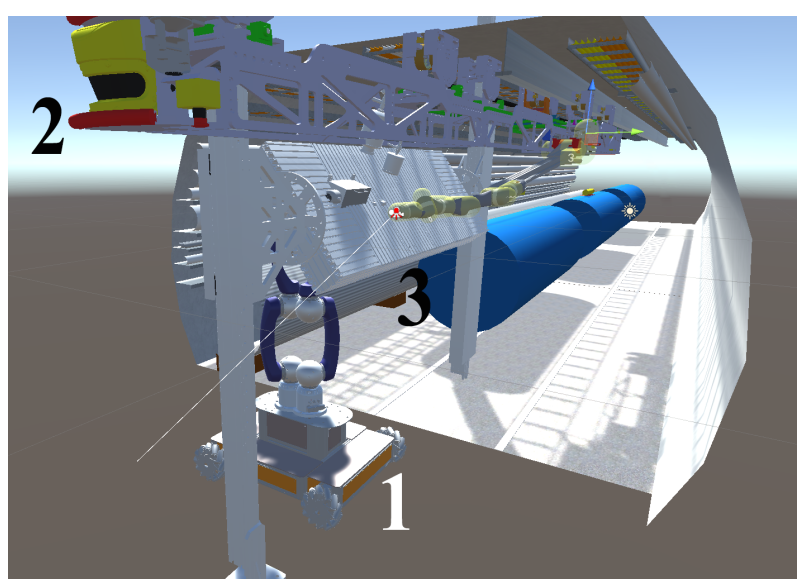

Figure 13: Bench simulation. The TIM (2) acts on the test bench (3) upstream side, which is installed next to the LHC mock-up, meanwhile CERNBot (1) acts on the its downstream side 


\section{CONCLUSIONS AND FURTHER WORK}

This paper has presented the current state of the design of a dynamic mock-up bench for testing robotic interventions. The bench offers a modular solution, enabling a realistic validation of heterogeneous operations.

Further steps will focus on the design of sensorized robotic tools and bench appliances (e.g. screws and connectors), which will allow the robots to perform repetitive tasks in a safe and autonomous manner. The sensors installed on the bench appliances will enable the use of machine learning algorithms to train the robots for autonomous operations.

\section{References}

[1] Lefevre, C. (2008). The CERN accelerator complex (No. CERN-DI-0812015).

[2] Di Castro, M., Buonocore, L. R., Ferre, M., Gilardoni, S., Losito, R., Lunghi, G., \& Masi, A. (2017, October). A dual arms robotic platform control for navigation, inspection and telemanipulation. In Proceedings of the 16th International Conference on Accelerator and Large Experimental Control Systems (ICALEPCS 2017), Barcelona, Spain (pp. 8$13)$.

[3] Di Castro, M., Tambutti, M. B., Ferre, M., Losito, R., Lunghi, G., \& Masi, A. (2018, August). i-TIM: A robotic system for safety, measurements, inspection and maintenance in harsh environments. In 2018 IEEE International Symposium on Safety, Security, and Rescue Robotics (SSRR) (pp. 1-6). IEEE.

[4] https://www.telerob.com/en/

[5] https://www.boschrexroth.com/en/us/prod ucts/product-groups/linear-motion-technolo gy/topics/linear-motion-systems/index

[6] https://www.tea-hamburg.de/katalog/img/ cms/Datenbl\%C3\%A4tter/Spindelhubgetrie be $\% 20$ mit\%20rotierender \%20Spindel $\% 20-\%$ 20Screw\%20Jacks\%20with\%20Rotating\%20 Screw.pdf

[7] https://harmonicdrive.de/de/produkt/antri ebe-mit-hohlwelle/canisdrive

[8] http://www.pandahoist.com/product/pamini-electric-hoist/

[9] Lunghi, G., Marin, R., Di Castro, M., Masi, A., \& Sanz, P. J. (2019). Multimodal human-robot interface for accessible remote robotic interventions in hazardous environments. IEEE Access, 7, 127290-127319.

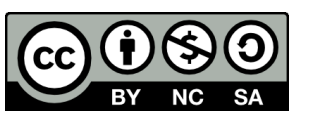
(C) 2021 by the authors. Submitted for possible open access publication under the terms and conditions of the Creative Commons Attribution CC BY-NC-SA 4.0 license (https://creativecommons.org/licenses/by-ncsa/4.0/deed.es). 